\title{
A Note On the Weak Convergence of the Extragradient Method for Solving Pseudo-Monotone Variational Inequalities
}

\section{Phan Tu Vuong ${ }^{1}$ (D)}

Received: 24 January 2020 / Accepted: 7 February 2020 / Published online: 2 March 2020

(c) The Author(s) 2020

\section{Abstract}

A corrigendum in Vuong (J Optim Theory Appl 176:399-409, 2018) is given.

Keywords Variational inequality · Extragradient method · Pseudo-monotonicity · Weak convergence

Mathematics Subject Classification 47J20 · 49J40 • 49M30

\section{Introduction}

The weak convergence of the extragradient method for solving pseudo-monotone variational inequalities in infinite dimensional Hilbert spaces was studied recently in [1]. The convergence analysis requires the sequential weak continuity of the associated operator. The illustrated example given in [1, Section 4] unfortunately does not satisfy this assumption. We provide in this note new examples satisfying all assumptions required.

\section{Updating of Reference 1}

Let $H=\ell_{2}$, the real Hilbert space, whose elements are the square-summable sequences of real numbers. Let $\beta>0$ and

$$
F_{\beta}(u):=(\beta-\|u\|) u, \quad \forall u \in H .
$$

$凶$ Phan Tu Vuong

T.V.Phan@soton.ac.uk

1 Mathematical Sciences, University of Southampton, University Road, Southampton SO17 1BJ, UK 
It is proved in [1] that the operator $F_{\beta}$ is pseudo-monotone and Lipschitz continuous. However, the sequential weak continuity of $F_{\beta}$ was not checked. Indeed, this assumption is not satisfied: let $\left\{e_{n}\right\}$ be the standard basic of $H$, i.e., $e_{n}=$ $(0, \ldots, 1,0, \ldots, 0)$ with 1 at the $n$-th position. Then $e_{1}+e_{n}$ converges weakly to $e_{1}$ but $F_{\beta}\left(e_{1}+e_{n}\right)=(\beta-\sqrt{2})\left(e_{1}+e_{n}\right)$ converges weakly to $(\beta-\sqrt{2}) e_{1}$ and $(\beta-\sqrt{2}) e_{1} \neq(\beta-1) e_{1}=F_{\beta}\left(e_{1}\right)$.

A correct example can be found in [2, Example 2.1], where the operator $F$ is pseudo-monotone, Lipschitz continuous and sequentially weakly continuous.

\section{Conclusions}

We give a corrigendum and new example to illustrate the main results obtained in [1]. The author thanks Radu Boţ for fruiful discussion in finding new examples and Heinz Bauschke for his awesome lectures at the University of Vienna in Dec, 2018, where the author learnt that the projection operator is not weakly continuous [3].

Open Access This article is licensed under a Creative Commons Attribution 4.0 International License, which permits use, sharing, adaptation, distribution and reproduction in any medium or format, as long as you give appropriate credit to the original author(s) and the source, provide a link to the Creative Commons licence, and indicate if changes were made. The images or other third party material in this article are included in the article's Creative Commons licence, unless indicated otherwise in a credit line to the material. If material is not included in the article's Creative Commons licence and your intended use is not permitted by statutory regulation or exceeds the permitted use, you will need to obtain permission directly from the copyright holder. To view a copy of this licence, visit http://creativecommons.org/licenses/by/4.0/.

\section{References}

1. Vuong, P.T.: On the weak convergence of the extragradient method for solving pseudo-monotone variational inequalities. J. Optim. Theory Appl. 176, 399-409 (2018)

2. Boţ, R.I., Csetnek, E.R., Vuong, P.T.: The forward-backward-forward method from continuous and discrete perspective for pseudo-monotone variational inequalities in Hilbert spaces. https://arxiv.org/ abs/1808.08084

3. Zarantonello, E.H.: Projections on Convex Sets in Hilbert Space and Spectral Theory. I. Projections on Convex Sets, Contributions to Nonlinear Functional Analysis, pp. 237-341. Academic Press, New York (1971). (Proc. Sympos., Math. Res. Center, Univ. Wisconsin, Madison, Wis., 1971)

Publisher's Note Springer Nature remains neutral with regard to jurisdictional claims in published maps and institutional affiliations. 